\section{Narrowing in on neurogenesis}

\section{By Tim Fulmer, Senior Writer}

Although aberrant neurogenesis in adults has been implicated in multiple CNS disorders, finding suitable targets for modulating the process without significant side effects has been difficult. As a result, the majority of drugs on the market and in development for neurological conditions act on signaling in adult neurons and not on the process by which neural stem cells actually proliferate, migrate and differentiate.

Now, a quartet of papers reveal new mechanisms by which two key signaling molecules-mTOR and GSK $3 \beta$ - could be targeted to either decrease or increase neurogenesis. ${ }^{1-4}$

Two of the papers show that inhibiting glycogen synthase kinase $3 \beta$ (GSK3 $\beta$ ) increases neurogenesis and could potentially be a strategy for treating schizophrenia and Alzheimer's disease. The second pair of papers suggests that inhibiting mammalian target of rapamycin (mTOR; FRAP; RAFT1) can decrease neurogenesis and thus potentially offer a therapeutic strategy for epilepsy and obsessivecompulsive disorder (OCD).

In all four papers, the researchers narrowed in on the two signaling molecules while looking for intracellular pathways that modulate transcription and synthesis of proteins potentially involved in neurogenesis. Because the two targets are essential to many healthy tissues throughout the brain and the rest of the body, two of the papers look at targeting signaling partners of mTOR and GSK $3 \beta$ rather than directly targeting the two proteins. This could offer a safety advantage by maintaining some basal levels of mTOR and GSK3 $\beta$ activity in healthy tissue.

\section{GSK3 $\beta$ in CNS disorders}

GSK3 $\beta$ has been implicated in CNS disease, ${ }^{5}$ but little is known about how the enzyme actually contributes to CNS pathology. Perhaps as a result, no GSK3 $\beta$-targeting compounds have been developed specifically for neuropsychiatric or neurodegenerative disorders.

Separate groups at the Massachusetts Institute of Technology and the Sun Health Research Institute have uncovered how aberrant upregulation of GSK3 $\beta$ leads to decreased neurogenesis and a disease phenotype (see Figure 1 "GSK3 $\beta$ in CNS disorders").

In a paper published in Cell, the MIT group reported that GSK3 $\beta$ was inhibited by disrupted in schizophrenia 1 (DISC1), a protein that is highly expressed in the adult hippocampus. ${ }^{6}$ Other researchers have shown that mutations in the DISC1 gene are associated

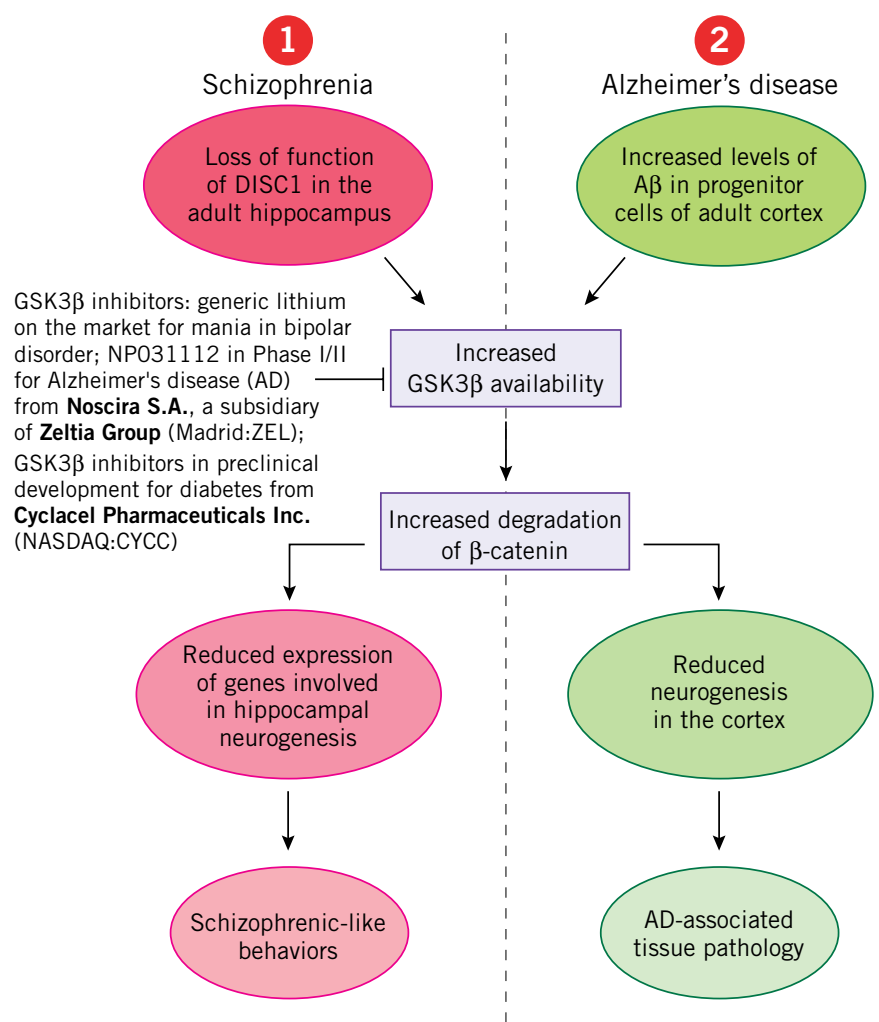

Figure 1. GSK3 $\beta$ in CNS disorders.

with schizophrenia risk, ${ }^{7}$ which led the authors of the Cell article to suggest that altered or absent DISC1 activity may contribute to schizophrenia in a GSK3 $\beta$-dependent manner.

Indeed, in the hippocampus of adult mice, small hairpin RNA knockdown of DISC1 led to increased GSK3 $\beta$ activity, increased degradation of the transcription factor regulator $\beta$-catenin and decreased proliferation of neural progenitor cells. The reduced proliferation of progenitors was associated with schizophrenic-like behavior, which was reversed with SB216763, a small molecule GSK3 $\beta$ inhibitor.

The compound was originally developed for cancer by SmithKline Beecham, now part of GlaxoSmithKline plc. The company did not return calls seeking comment on the compound's development status.

Li-Huei Tsai, corresponding author on the paper and a professor of neuroscience at MIT, told SciBX her next steps include developing DISC1 diagnostics and therapeutics.

Targeting DISC1 to block the GSK3 $\beta$-DISC 1 interaction could be a good idea "because questions of potential side effects will loom large with GSK $3 \beta$ inhibitors," said William Snider, director of the Neuroscience Center at The University of North Carolina at Chapel Hill School of Medicine. GSK3 $\beta$ is involved in the regulation of numerous cellular processes, including glycogen metabolism, cellcycle regulation and cellular proliferation. ${ }^{8,9}$

"Identification and characterization of rare and common genetic polymorphisms that may affect DISC1 function in humans will 


\section{TARGETS \& MECHANISMS}

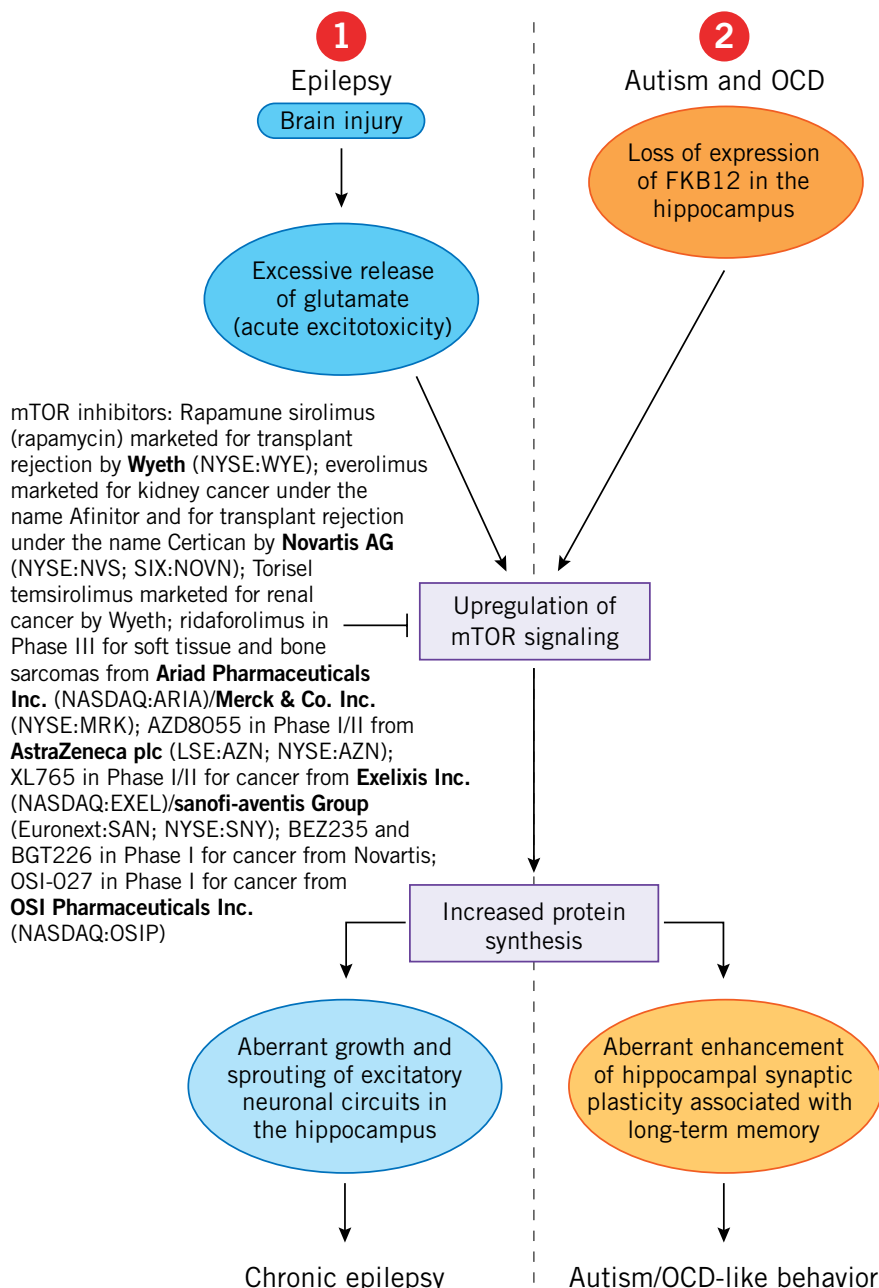

Figure 2. mTOR in CNS disorders.

provide further insight into the extent to which DISC1 is a risk factor for neuropsychiatric disease," said Tsai. "Gaining knowledge of the regulatory factors that govern the expression, trafficking and localization of DISC1 in neurons should help in the development of DISC1-based therapeutics."

MIT has filed a patent application covering the findings.

Meanwhile, Sun Health researchers have found that GSK3 $\beta$ activity was higher in glial progenitor cells isolated from the cortices of Alzheimer's patients than in cells from healthy controls. Glial progenitor cells in $\mathrm{AD}$ patients also had higher $\beta$-amyloid (A $\beta$ ) levels and a lower capacity for neurogenesis.

Transgenic mice overexpressing A $\beta$ had higher GSK3 $\beta$ activity, higher rates of $\beta$-catenin degradation and lower expression of several neurogenesis-related genes than wild-type mice.

The paper's findings suggest that increased $A \beta$ upregulates GSK3 $\beta$, which in turn reduces neurogenesis. The authors concluded that "approaches to inhibit GSK3 $\beta$ and/or elevate $\beta$-catenin in glial progenitor cells or other neural progenitor cells may allow us to mitigate the toxic effects of $A \beta$ and promote neurogenesis in $A D$ patients."
Yong Shen, corresponding author on the paper and senior scientist at Sun Health, told SciBX he is now interested in developing a strategy for gene delivery to improve neurogenesis in $\mathrm{AD}$ patients. None of the paper's findings is patented.

\section{More out of mTOR}

Although mTOR inhibitors like Rapamune sirolimus (rapamycin) are marketed as immunosuppressants and have been studied extensively in cancer, two other new papers suggest that inhibiting the target in the adult hippocampus could block aberrant neuronal growth and potentially help treat epilepsy and psychiatric disorders (see Figure 2, “mTOR in CNS disorders").

In an article published in The Journal of Neuroscience, Washington University in St. Louis School of Medicine researchers reported increased mTOR activity in the mouse hippocampus following chemically induced brain seizure. The mTOR activity was associated with long-term increases in protein synthesis and eventual development of chronic epilepsy.

The researchers therefore hypothesized that inhibiting mTOR might help treat or prevent recurrent epileptic seizures. Indeed, administration of rapamycin in the mice following the initial brain seizure blocked development of chronic recurrent epileptic seizures and reduced the growth of hippocampal mossy fibers that can give rise to excitatory neuronal circuits and cause long-term epilepsy.

"Following brain injury or trauma, there's typically a latent period before spontaneous recurrent epileptic seizures set in," said Michael Wong, corresponding author on the paper. "If mTOR inhibitors were administered during that latent period, they could perhaps help prevent epileptogenesis," the chronic condition of recurrent spontaneous seizures.

To better evaluate mTOR inhibition as a preventative strategy, Wong plans to test rapamycin in rodent models of trauma-induced epilepsy. In the longer term he hopes to study mTOR inhibitors in traumatic brain injury patients and in other people at high risk of epilepsy.

Wong is assistant professor of neurology, pediatrics, anatomy and neurobiology at the Washington University in St. Louis School of Medicine. None of the paper's findings is patented.

Whereas Wong and colleagues plan to directly target mTOR to prevent seizures, researchers at New York University and Baylor College of Medicine think indirectly modulating mTOR could treat some learning disorders.

The team observed that FK506-binding protein 12 (FKBP12) knockout mice showed autistic and OCD-like behavior in several tasks. FKBP12 is an intracellular modulator of mTOR activity. The knockouts also had increased mTOR activity in the hippocampus, which led to increased hippocampal long-term potentiation and greater synaptic plasticity.

The authors concluded that FKBP12 may suppress mTOR signaling and hold in check aberrant hippocampal synaptic plasticity that could give rise to autism and OCD. The results were published in Neuron.

The paper "adds to the growing notion that mTOR signaling may be a useful intervention point for therapeutic approaches to treating cognitive disorders," said Michael Sutton, assistant professor of molecular and integrative physiology at the University of Michigan. 


\section{TARGETS \& MECHANISMS}

However, Sutton added, inhibiting a ubiquitous molecule like mTOR could have many adverse side effects. The findings reported in the Neuron article offer the potentially safer alternative of targeting a modulator of mTOR signaling rather than hitting mTOR itself. By doing this, some level of essential mTOR signaling may remain intact, he told SciBX.

The label for Rapamune, the mTOR inhibitor marketed by Wyeth to prevent organ rejection in renal transplant patients, states that side effects include peripheral edema, hypertension and increased susceptibility to infection and malignancy. Wyeth is being acquired by Pfizer Inc.

Eric Klann, corresponding author on the paper and professor of neuroscience at NYU, told SciBX he is interested in developing compounds that mimic the actions of FKBP12 on mTOR. None of the paper's findings is patented.

Fulmer, T. SciBX 2(23); doi:10.1038/scibx.2009.930

Published online June 11, 2009

\section{REFERENCES}

1. Mao, Y. et al. Cell; published online March 20, 2009; doi:10.1016/j.cell.2008.12.044

Contact: Li-Huei Tsai, Massachusetts Institute of Technology, Cambridge, Mass.

e-mail: Ihtsai@mit.edu

2. He, P. \& Shen, Y. J. Neurosci.; published online May 20, 2009;
doi:10.1523/JNEUROSCI.0421-09.2009

Contact: Yong Shen, Sun Health Research Institute, Sun City, Ariz. e-mail: yong.shen@bannerhealth.com

3. Zeng, L.-H. et al. J. Neurosci.; published online May 27, 2009; doi:10.1523/JNEUROSCI.0066-09.2009

Contact: Michael Wong, Washington University in St. Louis School of Medicine, St. Louis, Mo.

e-mail: wong_m@wustl.edu

4. Hoeffer, C. et al. Neuron; published online December 11, 2008; doi:10.1016/j.neuron.2008.09.037

Contact: Eric Klann, New York University, New York, N.Y. e-mail: eklann@cns.nyu.edu

5. Jope, R. \& Roh, M.-S. Curr. Drug Targets 7, 1421-1434 (2006)

6. Duan, X. et al. Cell 130, 1146-1158 (2007)

7. Chubb, J. et al. Mol. Psychiatry 13, 36-64 (2008)

8. Doble, B. \& Woodgett, J. J. Cell Sci. 116, 1175-1186 (2003)

9. Cohen, P. and Frame, S. Nat. Rev. Mol. Cell Biol. 2, 769-776 (2001)

COMPANIES AND INSTITUTIONS MENTIONED

Baylor College of Medicine, Houston, Texas

GlaxoSmithKline plc (LSE:GSK; NYSE:GSK), London, U.K.

Massachusetts Institute of Technology, Cambridge, Mass.

New York University, New York, N.Y.

Pfizer Inc. (NYSE:PFE), New York, N.Y.

Sun Health Research Institute, Sun City, Ariz.

University of Michigan, Ann Arbor, Mich.

The University of North Carolina at Chapel Hill School of

Medicine, Chapel Hill, N.C.

Washington University in St. Louis School of Medicine, St. Louis, Mo. Wyeth (NYSE:WYE), Madison, N.J. 